E-Business und Nachhaltigkeit

\title{
Strategiefelder der Zukunft
}

Die anfängliche Euphorie über die New Economy ist nach dem Scheitern zahlreicher Internet-Startups und angesichts des nach wie vor geringen Umsatzanteils von E-Commerce einer realistischeren Einschätzung gewichen. E-Business ist auch in Zukunft nicht alles, Internetnutzung und E-Commerce werden allerdings an Bedeutung gewinnen. Mit Blick auf die Ziele einer nachhaltigen, umweltverträglichen Entwicklung sind die zu erwartenden Effekte allerdings ambivalent. Wo liegen für Unternehmen strategische Ansatzpunkte, um die Potenziale der Internetrevolution für den Umweltschutz zu nutzen?

$\mathrm{D}$ Kaus Fichter ie Umwelteffekte von Informations- und Kommunikationsgeräten und -techniken sind bereits seit einer Reihe von Jahren Gegenstand umweltpolitischer Diskussionen und betrieblicher Entwicklungsanstrengungen. Die Frage, wie durch die Nutzung von Internet und Informations- und Kommunikationstechnologien (IuK-Technologien) ein aktiver Beitrag zu einer nachhaltigen Entwicklung geleistet werden kann, findet hingegen bis dato vergleichsweise wenig Beachtung. Eine Ausnahme bildet hier die Debatte um Telematikanwendungen im Verkehrsbereich (1).

Bislang sind Umweltentlastungen durch die Nutzung von Internet und E-Business in der Regel nicht-intendierte zufällige Nebeneffekte, da Telekommunikation und Internet vorrangig zur Optimierung von Geschättsabläufen und zur Sicherung oder Erschließung von Märkten genutzt werden (2). Der wirtschaftliche und ökologische Bedeutungszuwachs von E-Business und Internet machen für die Zukunft aber eine gezielte Berücksichtigung von Umweltanforderungen im Rahmen von Unternehmensstrategien notwendig. Grundsätzlich können hier vier Strategiefelder unterschieden werden (siehe auch Abbildung 1):

- Greening of IT: Umweltverträgliche Gestaltung und Nutzung von IuK-Geräten und -techniken.

- E-Substitution: Substitution physischer Produkte durch elektronische, umweltverträglichere Produkt-, Versand- und Nutzungsalternativen.

- E-Support: Nutzung von Internet und E-Business zur Unterstützung und Verbreitung umweltschonender Produkte und Dienstleistungen.
E-Services: Nutzung internetgestützter Dienstleistungen, Geschäftsmodelle und Netzwerke zur Umweltentlastung und Verbesserung der Öko-Effizienz.

Innovationsstrategien für die umweltverträgliche Gestaltung und Nutzung von IuK-Techniken wurden an anderer Stelle bereits ausführlich diskutiert (3). Das Strategiefeld Greening of IT wird daher im folgenden nicht weiter betrachtet.

\section{E-Substitution}

Der Strategieansatz der elektronischen Substitution physischer Güter - wie Briefe, Telefonbücher, Zeitungen, Konstruktionsunterlagen - und von Transporten ist von seiner Idee her nicht neu. Allerdings zeigt sich, dass die Umweltentlastungspotenziale dieses Ansatzes bislang kaum untersucht sind und in der Unternehmenspraxis bis dato selten systematisch genutzt werden. Der Ansatz wirft die grundsätzliche Frage auf, ob elektronische Produkt- oder Versandalternativen über den gesamten Produktlebenszyklus betrachtet tatsächlich umweltschonen- der sind. Da es hier bislang kaum belastbare Daten und keine generellen Antworten gibt, muss dies im Einzelfall abgeschätzt werden. Für diese Zwecke dürfte aufgrund der Komplexität des Untersuchungsobjektes eine vollständige Produkt-Ökobilanzierung in der Regel zu aufwendig sein. Einfachen, aber richtungssicheren Methoden wie dem Konzept des kumulierten Energieaufwandes ist hier aus Kosten-Nutzen-Erwägungen wohl zumeist der Vorzug zu geben.

\section{E-Support}

Größere Umweltentlastungspotenziale dürften in der Internetunterstützung bei der Leistungserstellung und Produktnutzung liegen (E-Support). Dies gilt zum Beispiel für die Verbesserung der Kommunikation im Rahmen einer kundenspezifischen und öko-effizienten Massenproduktion. Ein Beispiel hierfür ist die Firma ChemStation aus Ohio, die mit Hilfe des Internets kundenspezifische Reinigungsmittel herstellt und vertreibt und damit unnötige Zusatzstoffe oder eine falsche Dosierung vermeidet (4). Interessant sind auch andere Ansätze kundenspezifischer Produktion wie z.B. „Print-on-demand“- und „Build-to-order“-Strategien (5).

Eine interessante Form, physische Produkte durch Internetunterstiutzung besser zu nutzen, besteht in der Verwendung von Transpondern (,smart tags"), die neben einem einfachen Mikrochip auch eine Sende- und Empfangseinheit enthalten und damit über das Internet „kommunizieren“ können. Der hauchdünne Transponder lässt sich in Produkte, etwa Hausgeräte, oder in Verpackungen integrieren. Aufgrund des gesunkenen Stïckpreises ist der Einsatz wirtschaftlich mittlerweile interessant (6). Mit der Internetfähigkeit von Produkten ergeben sich neue Möglichkeiten, die Produktnutzung, die Produktlebensdauer und das Recycling zu verbes-

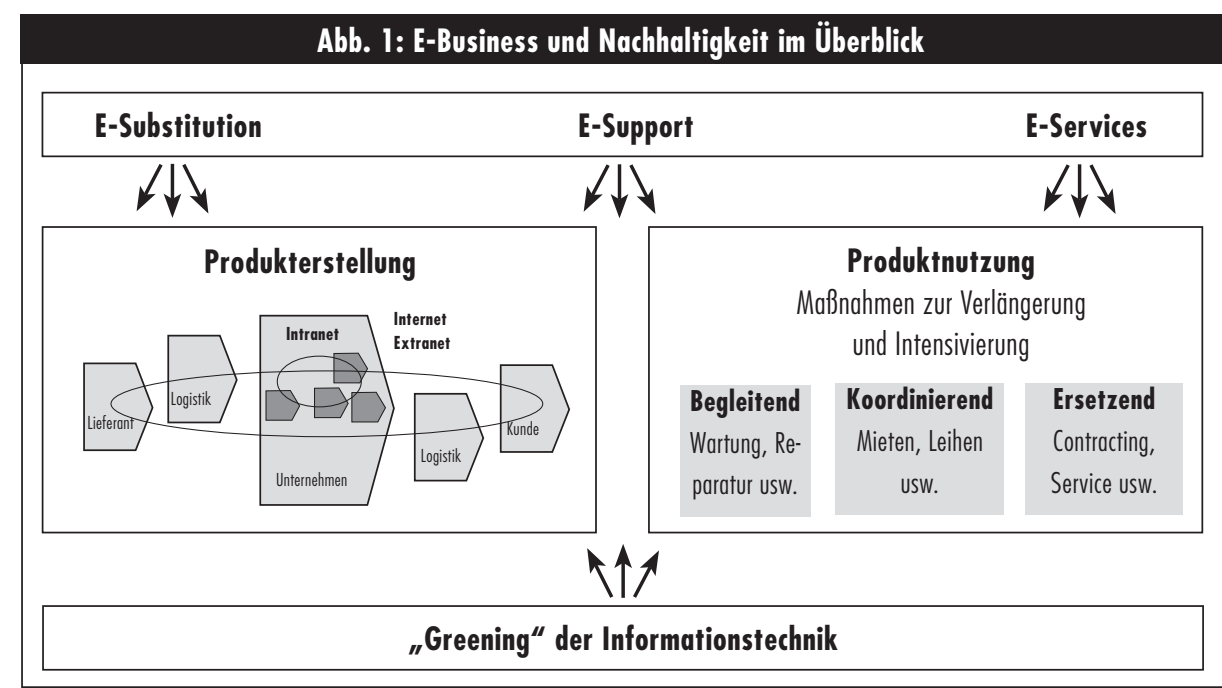


sern. Produktreklamationen und -wartungen werden erleichtert, aktuelle Sicherheitsinformationen zu Produkten können einfacher übermittelt und neue Anreize zur sparsamen Nutzung von Produkten entwickelt werden. So testet beispielsweise Electrolux derzeit internetfähige Hausgeräte, die an Verbraucher vermietet und nach Gebrauch bezahlt werden. Damit können Anreize zur effizienten Nutzung geschaffen werden.

\section{E-Services}

Ein weiterer bedeutsamer Strategieansatz für nachhaltige E-Business-Lösungen liegt in der Entwicklung internetgestïtzter Dienstleistungen und Unternehmensnetzwerke (E-Services). So können beispielsweise berufs- und funktionsspezifische Internetportale, die neueste Branchen- und Technologieinformationen anbieten, von Entwicklungsingenieuren oder Innovationsmanagern für die Suche nach umweltschonenden Technologien und Servicemodellen genutzt werden. Neue Möglichkeiten des Stoffstrommanagements entstehen durch den Einsatz des Internets. So hat die Firma Henkel eine internetgestïtzte Reststoffbörse eingerichtet, bei der in den Produktionsbetrieben nicht mehr einsetzbare Rest-Rohstoffe, Nebenprodukte und Fehlchargen systematisch zur Weiterverwendung angeboten werden. Auf diese Weise konnte Henkel 1999 Rohstoffe im Umfang von 4.150 Tonnen und im Wert von 2,8 Millionen Mark einsparen (7). Auch im Bereich der Produktnutzungsverlängerung und des Produktrecyclings können durch internetgestïtzte Dienstleistungen neue Umweltentlastungspotenziale erschlossen werden. Ein Beispiel hierfür ist die Auto-Recyclingbörse im Internet der Firma renet (8). Beide Beispiele wären ohne die Senkung der Transaktionskosten (Suchkosten, Vereinbarungskosten usw.) durch das Internet nicht möglich.

\section{Fazit}

Internet und E-Commerce sind als Medium und Markttransaktionsform der Zukunft von zentraler Bedeutung für eine nachhaltige Entwicklung. Bislang sind drei Dinge klar:

1. Belastbare Daten und Fakten über die Umweltwirkungen von E-Commerce und Internetnutzung liegen kaum vor. Hier herrscht erheblicher Forschungsbedarf.

2. Plausible Annahmen führen zu der Prognose, dass der Einsatz neuer IuK-Technologien sowohl umweltentlastende wie auch umweltbelastende Effekte aufweisen wird, ohne dass das ökologische Gesamtergebnis per saldo schon absehbar ist (9).
3. Trotz der hohen Eigendynamik bei der Entwicklung von IuK-Technologien und der rasanten $\mathrm{Zu}$ nahme der Internetnutzung ist die digitale Revolution nicht deterministisch, sondern in vielen Bereichen zukunftsoffen und damit gestaltbar. Nicht das Medium (Internet) bestimmt die Nachhaltigkeit, sondern die umweltschonende und intelligente Gestaltung.

Neben der Schaffung geeigneter politischer Rahmenbedingungen kommt es auf die intelligente Nutzung von Internet und E-Business durch Unternehmen an. Dabei gilt es im Rahmen des Innovationsmanagements die Umweltbelastungen von IuKTechniken zu minimieren und bei Geschäftsmodellen des E-Business und internetgestützten Produkten und Dienstleistungen Umwelteffekte frühzeitig zu berücksichtigen.

\section{Anmerkungen}

(1) Vgl. die IZT-Studie von Gaßner, R. et al.: Telematik und Verkehr - Elektronische Wege aus dem Stau? Weinheim 1994. (2) Öko-Institut e.V. (Hrsg.): Umweltschutz im Cyberspace, Zur Rolle der Telekommunikation für eine nachhaltige Entwicklung, Freiburg 1997

(3) Vgl. Behrendt, S./ Pfitzner, R./ Kreibich, R./ Hornschild, K.: Innovationen zur Nachhaltigkeit - Ökologische Aspekte der Informations- und Kommunikationstechnik,

Berlin u.a. 1998.

(4) Vgl. im Internet unter www.chemstation.com

(5) Vgl. Cohen, N.: E-Commerce and the Environment, in: Tellus Institute (Hrsg.): Environmental Perspectives, Special Edition "Environment and the Information Age", April 2000, Boston, S. 2-5 (Internet: www.tellus.org).

(6) Vgl. im Internet unter http://auto-id.mit.edu

(7) Vgl. Rauberger, R./ Wisser, J.: Die Reststoffbörse bei Henkel: Rohstoffeinsparungen durch Nutzung von luK-Technologien, in: Fichter, K. / Schneidewind, U. (Hrsg.): Umweltschutz im globalen Wettbewerb, Berlin u.a. 2000,

S. 333-338.

(8) Vgl. Albrecht, B.: Auto-Recyclingbörsen im Internet, in: Fichter, K./Schneidewind, U. (Hrsg.): Umweltschutz im globalen Wettbewerb, Berlin u.a. 2000, S. 327-331.

(9) Vgl. Fichter, K.: Nachhaltige Unternehmensstrategien in der Internet-Ökonomie, in: Schneidewind, U./ Steingräber, G./ Truscheit, A. (Hrsg.): Nachhaltige Informationsgesellschaft, Berlin u.a. 2000, S. 69-83.

\section{Der Autor}

Dr. Klaus Fichter ist Geschäftsführer des Institutes für Innovations- und Nachhaltigkeits-Forschung (INF) und leitete von 1993 bis 1999 das IÖW-Forschungsfeld Ökologische Unternehmenspolitik. Kontakt: Lyckallee 26, 14055 Berlin, Tel. 030/ 34231 04, Fax 030/ 301085 55, E-Mail: fichter@snafu.de

\section{politische ökologie a-z}

\section{Wählen Sie aus unserem Gesamtverzeichnis}

pö 51 Zukunftsaufgabe Umweltbildung - Auf der Suche nach neuen Perspektiven

pö 52 Geduldsspiel Nachhaltigkeit -Agenda 21 als Leitfaden für das nächste Jahrhundert

pö 53 Wechsel ohne Deckung - Über den Zusammenhang von Geld und Umwelt

pö 54 Nachhaltiges Arbeiten - Ein Weg aus der Beschäftigungs- und Umweltkrise?

pö 55 Gratwanderung - zwischen Autarkie und Clobalisierung: Die Alpen als Vorreiter für ein regionales Wirtschaften

pö 56 Endspurt - Die Ökologische Steuerreform vor dem Durchbruch?

pö 57/58 Von der Zeitnot zum Zeitwohlstand Auf der Suche nach den rechten Zeitmaßen

pö 59 Wa(h)re Wildnis - Reise Special ' 99

pö 60 Risiko

pö 61 Wechselstrom - Anleitung für eine neue Energiepolitik

pö 62 Stoffwechsel - Naturstrategien als Vorbild für Nach-haltigkeit

pö 63/64 Nachhaltigkeit öffne dich! Kommunikation eines Leitbildes inklusive Literatur Spezial 2000 pö 65 e_cotopia - von ökologischen visionen zu virtuellen realitäten

(Einzelhefte 19,80 DM, Doppelausgaben 24,80)

\section{Sonderhefte}

pö Sonderheft 8 Zeit-Fraß - Zur Ökologie der Zeit in Land-wirtschaft und Ernährung

pö Sonderheft 9 Handwerk als Schlüssel für eine zukunftsfähige Wirtschaft

pö Sonderheft 10 Bodenlos - Zum nachhaltigen Umgang mit Böden

pö Sonderheft $\mathbf{1 1}$ Wege aus der Wachstumsfalle - Perspektiven einer zukunftsfähigen Wirtschaftsund Lebensweise

pö Sonderheft 12 Schnittmenge Mensch - Bildung für nachhaltige Entwicklung als neue Lernkultur

(Sonderhefte zum Preis von je 19,80 DM)

PÖ-Reise-Special 2000 - Exponierte Zukunft 12,80 DM

PÖ-Literatur Special - Umweltbücher 2000 $12,80 \mathrm{DM}$

Abo für sechs Ausgaben: DM 98,00, (Institutionen DM 148,-), ermäßigt: DM 78,00 (ohne Versandkosten)

Eine Übersicht aller noch erhältlichen Ausgaben finden

Sie auch auf unserer Internetseite:

www.oekom,de 
(c) 20I0 Authors; licensee IÖW and oekom verlag. This is an article distributed under the terms of the Creative Commons Attribution Non-Commercial No Derivates License (http://creativecommons.org/licenses/by-nc-nd/3.o/), which permits unrestricted use, distribution, and reproduction in any medium, provided the original work is properly cited. 